\title{
On the Optimal Density for Real-Time Data Gathering of Spatio-Temporal Processes in Sensor Networks
}

\author{
Răzvan Cristescu \\ Caltech, 136-93 Pasadena CA 91125, USA \\ razvancecal tech.edu
}

\author{
Martin Vetterli \\ EPFL, Lausanne CH-1015, Switzerland \\ UC Berkeley, CA 94720, USA \\ Martin.Vetterli@epfl.ch
}

\begin{abstract}
We consider sensor networks that measure spatio-temporal correlated processes. An important task in such settings is the reconstruction at a certain node, called the sink, of the data at all points of the field. We consider scenarios where data is time critical, so delay results in distortion, or suboptimal estimation and control. For the reconstruction, the only data available to the sink are the values measured at the nodes of the sensor network, and knowledge of the correlation structure: this results in spatial distortion of reconstruction. Also, for the sake of power efficiency, sensor nodes need to transmit their data by relaying through the other network nodes: this results in delay, and thus temporal distortion of reconstruction if time critical data is concerned. We study data gathering for the case of Gaussian processes in one- and twodimensional grid scenarios, where we are able to write explicit expressions for the spatial and time distortion, and combine them into a single total distortion measure. We prove that, for various standard correlation structures, there is an optimal finite density of the sensor network for which the total distortion is minimized. Thus, when power efficiency and delay are both considered in data gathering, it is useless from the point of view of accuracy of the reconstruction to increase the number of sensors above a certain threshold that depends on the correlation structure characteristics.
\end{abstract}

\section{INTRODUCTION}

\section{A. Motivation}

Consider a sensor network measuring a data field. The measured process is a spatio-temporal correlated process. Namely, the value corresponding to a certain coordinate $x$ in the measured area depends both on the values measured at the other nodes, and on the previous values measured at point $x$. Typical examples of correlated processes include various environment quantities (e.g. temperature, pressure, humidity, particle concentrations). In most practical settings, the correlation of the process decreases with both time and the distance between nodes.

One important task in such a scenario is data gathering and estimation, where a central node $S$, called the sink, needs to estimate the data in the whole field, with a certain minimum distortion. For this, a fixed number $N$ of nodes are placed in the field (see Fig. 1). These nodes form an interconnection structure which is usually an undirected connected graph. Nodes transmit their measurements to the sink, at given time instants, by using a subset of the links of the graph. The sink needs to reconstruct the whole field with a minimal total distortion. We consider settings where data is time critical, and thus delay results in distortion. Such settings include scenarios for fire prevention, or seismic awareness; extremal settings include sensor networks measuring phenomena where abrupt transitions are critical (e.g. cracks in a massive structure, or mudslides over a large terrain area). Another class of relevant scenarios is when sink feedback or control is needed at nodes, and where the effect of delay in reporting the data induces suboptimal estimation and control.

If the network is dense (large $N$ ), the data has a good spatial approximation. However, for power efficiency reasons, nodes in sensor networks cannot transmit their data directly to the sink, but

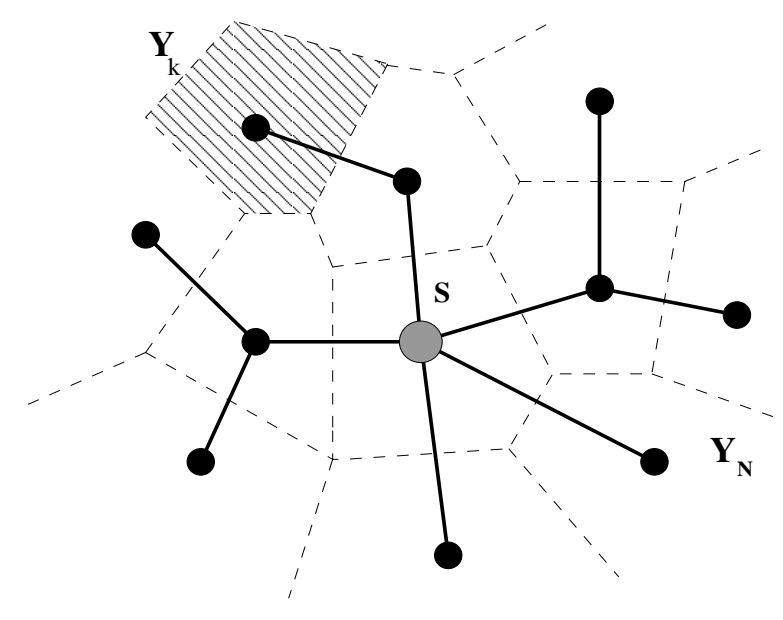

Fig. 1. In this example, spatio-temporally correlated data from nodes $1, \ldots, N$ need to arrive at sink $S$. The sink needs to reconstruct the whole data field using only the measured values $Y_{1}, \ldots, Y_{N}$. Arbitrary points in the two-dimensional space are approximated by the measurements of the sensor node corresponding to the Voronoi cell to which they belong. The dashed zone corresponding to node $k$ represents the area of the field approximated by the value $Y_{k}$ in node $k$. In thick solid lines, a chosen transmission structure is shown (here, the shortest path tree $S P T$ ). Data from node $k$ reaches the sink after being relayed by one other sensor node.

rather communication is usually done via multihopping. This implies long delays until the data sent from nodes far from the sink reach the sink, which thus results in weak temporal approximation.

On the contrary, opposite effects take place when $N$ is small. Namely, the spatial approximation of the data is poor, but on the other hand data has to travel over only a limited number of hops, which results in a good temporal approximation. Thus, as we will show in this paper, for a given spatio- temporal correlation structure, there usually exists a finite optimal $N$ that minimizes the overall distortion of the field reconstruction at the sink.

\section{B. Problem Setup}

We study the influence of node density on the total distortion of estimation, when several aspects specific to sensor nodes are considered, namely delay and energy efficiency. Our setting takes into account two important issues typical in sensor networks scenarios: the precision of estimation, given by the density of the sensor network deployed to measure the field [8], and the energy efficiency, which results in the necessity of multihopping the data [10].

First, since the measured data is correlated and the number of available nodes is limited, the sink can reconstruct the values of the field at each point by approximating them with the values at the points where the actual sensor nodes are placed. In those points, full 
measurements are available. Also, no other information except the values measured at sensor nodes is available at the sink about that region of the field. The precision of the approximation depends both on the level of spatial correlation in the data and on the number of sensors available. This approximation introduces a first factor of distortion, which we call 'spatial distortion'.

Second, since the nodes have limited battery power, a good strategy is to send data via relaying nodes rather than directly to the sink (multihopping). However, multihopping results in data delay, since data from the extremities of the network need to be transmitted via multiple relays until they reach the sink. In various practical sensor network scenarios, data is needed at the sink in real-time. For instance, for the tasks of control or active monitoring, data may become useless if it arrives at the sink with a too large delay. For a spatio-temporal correlated process, the data that arrive at the sink are distorted from the original measured values, however they can be reconstructed with a certain precision given by the intensity of temporal correlation of the process. Thus, delay introduces a second factor of distortion, which we call 'time distortion'.

In our study, the two types of distortion are modelled as a single distortion per field point quantity. Their combined effect results in the total distortion of the field at the sink, and the goal of this paper is to study how this quantity is influenced by the density of nodes of the sensor network. Namely, we argue that for various typical spatio- temporal correlation models of the data field, there is a unique optimal value for the number of placed nodes $N$ that minimizes the total spatio-temporal distortion.

To the best of our knowledge, this is the first research work that consider both spatial estimation and delay in data gathering sensor networks, in terms of a single meaningful quantity to be optimized, namely total distortion.

For the sake of simplicity, in this work we analyze one-dimensional and two-dimensional regular grid settings. In our study, we consider Gaussian random processes which exhibit spatio-temporal correlation. The general setting is a subject of our current research, and we believe the insights provided in this paper are valid for more general network scenarios and structures in the correlation of the data.

\section{Related Work}

Efficient data gathering of spatially correlated data has been studied in [4], [3], [6]. An analysis of the impact of data irregularity on the spatio-temporal sampling is done in [5]. Our novel take on the problem of data gathering of spatio-temporal processes is that we are able to formulate the problem in terms of a unique performance measure, namely the total distortion.

\section{Main Contributions and Organization of the Paper}

The main contribution of this paper is an analysis of real-time data gathering of spatio-temporally correlated processes in multihop sensor networks, in terms of a single performance measure, namely the distortion of reconstruction. We are able to show both theoretically and experimentally that for the class of widely used Gaussian correlated processes, in many cases there exists an optimal finite density of the network for real-time data gathering. Namely, when both delay and power efficiency are considered, the optimal network size to sample a given fixed size area is finite ${ }^{1}$ and increasing the number of nodes above this threshold deteriorates the distortion of

\footnotetext{
${ }^{1}$ Note that the Gaussian processes studied in this paper are not bandlimited. Similar results will hold for bandlimited processes sampled above Nyquist frequency, since their correlations are fastly decreasing with time and distance.
}

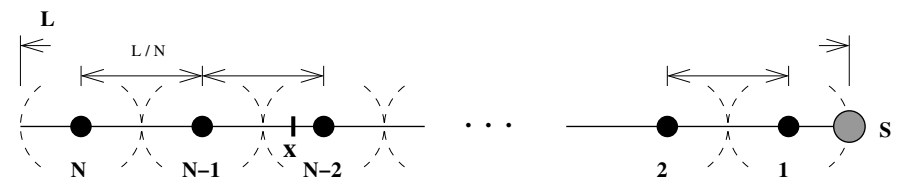

Fig. 2. A one-dimensional example of a data gathering network, where each sensor node covers an area of the whole network. An instantaneous point of reconstruction is denoted by $x$. In this example, $x$ belongs to the Voronoi cell of node $N-2$, thus its value at time $t$ is approximated, with a certain distance dependent distortion, by $Y\left(x_{N-2}, t\right)$.

reconstruction. The analysis in this paper is restricted to one- and two-dimensional grid networks, but we expect our results to hold for more general distributed networks.

Section II introduces the transmission model and signal model we use in this paper. Further, a performance measure of interest is defined, namely the total distortion, and an analysis of this measure for the one-dimensional grid is performed. Section III generalizes the model in Section II for a two-dimensional grid. In Section IV we present our numerical simulations, and we conclude with Section V.

\section{ONE-DimEnSIONAL NETWORK}

\section{A. Transmission Model}

For simplicity of the analysis, in this paper we consider networks with nodes uniformly placed on one- and two-dimensional grids, for which the distortion optimization is done only with respect to the size of the network. In the case of arbitrary networks with positiondependent correlation structure, the optimization becomes a function of the node placement as well.

Consider $N$ nodes placed on a line of fixed length $L$ (see Fig. 2). The inter-node distance is $d=L / N$. An additional node $S$ at the extreme right of the line represents the sink to which all data should arrive. The task of the sink is to reconstruct the whole field on the line.

We assume that the quantization done at nodes is very fine, namely we assume the reconstruction error at the sink is only an estimation error. For that, points on the line that belong to the space intervals among the $N$ nodes are assigned to Voronoi cells of the sensor nodes; these cells are delimited by mid-interval points. Therefore, each sensor node covers an interval of length $d$ around its position. The values of the intermediate points are estimated at the sink by the value of the corresponding sensor node in the middle of the Voronoi cell $^{2}$ (see Fig. 2). In this work, we assume that the measurements at nodes have the same variance, and that the rates allocated at nodes are equal. This implies that nodes use equal transmission power, and that the spatial distortion per cell does not depend on the node identity. The study of networks with different rates at nodes is a straightforward generalization, and is a subject of our current research.

For the sake of power efficiency, as mentioned in Section I-B, nodes need to relay their data. Namely, they need to send their data to the sink by multihopping via nodes on the corresponding path on the transmission structure that links them to the sink. This inherently introduces delay, since data that travels through the network reaches the sink at a later time than the time at which it was measured. The delay has two causes. On the one hand, if relaying nodes have finite

\footnotetext{
${ }^{2}$ More complex estimation strategies in which the field value at an arbitrary position is based on the measurements of more than a single sensor node are a subject of our current investigations [9], [7].
} 
buffers then packet forwarding results in a certain processing time due to buffering, and thus the delay is proportional to the number of hops that data needs to travel. On the other hand, if communication edges are lossy channels then this requires retransmissions of data, and thus the delay is proportional to the number of edges that data needs to travel. For the sake of simplicity, we assume that the delay is proportional to the number of hops. Therefore, since the measured process is correlated both in time and in space, the reconstruction is further distorted from the real-time value due to delay caused by relaying.

\section{B. Signal Model}

We assume a spatio-temporal correlated process $Y(x, t)$, where $x$ denotes the space dimension, and $t$ denotes the time dimension. We further assume that the process measured by the field is Gaussian distributed Namely, each node measures a zero-mean and unit variance normal random variable $Y(x, t) \approx \mathcal{N}(0,1)$, which is correlated both in space and in time with the rest of the network nodes. We consider correlation structures of the form

$$
\begin{aligned}
E\left[Y\left(x_{1}, t_{1}\right) Y\left(x_{2}, t_{2}\right)\right] & =\sigma_{Y\left(x_{1}, t_{1}\right), Y\left(x_{2}, t_{2}\right)} \\
& =\sigma\left(\left|x_{1}-x_{2}\right|,\left|t_{1}-t_{2}\right|\right) \\
& =e^{-\alpha\left(\left(x_{1}-x_{2}\right)^{2}+\beta^{2}\left(t_{1}-t_{2}\right)^{2}\right)^{\kappa}},
\end{aligned}
$$

with $\beta$ the scaling constant for the time axis, and $\kappa=\frac{1}{2}$ corresponding to a Gauss-Markov field or $\kappa=1$ corresponding to a squared distance correlation model, and $\alpha$ a constant that measures the intensity of correlation [2]. Note that this is a generalization of the space correlation models used in [4], with one of the spatial dimensions replaced by a scaled time dimension.

For instance, consider the value $Y\left(x, t_{0}\right)$ of an arbitrary point $x$ on the line at an arbitrary time $t_{0}$ (see Fig. 3). Assume that the sink approximates the value at point $x$ by considering the value $Y\left(x_{0}, t_{0}\right)$, at point $x_{0}$, placed $k$ hops away from the sink. For any data packet, we assume that the relation between the time delay $t_{k}$ of that packet and the number of hops $k$ it has to travel is $k=\beta t_{k}$. Assume the delay per hop is a constant $T$, thus $t_{k}=k T$. Then, the mean-square error (MSE) of $Y\left(x, t_{0}\right)$ at the sink, when $Y\left(x_{0}, t_{0}\right)$ is known, is expressed by:

$$
\begin{aligned}
D_{x, t_{0}, x_{0}, k}= & E\left[\left(Y\left(x, t_{0}\right)-Y\left(x_{0}, t_{0}+k T\right)\right)^{2}\right]- \\
= & E\left[Y\left(x, t_{0}\right)^{2}\right]+E\left[Y\left(x_{0}, t_{0}+k T\right)^{2}\right] \\
& -2 E\left[Y\left(x, t_{0}\right) Y\left(x_{0}, t_{0}+k T\right)\right] \\
= & 2-2 \sigma_{Y\left(x, t_{0}\right) Y\left(x_{0}, t_{0}+k T\right)} \\
= & 2-2 \sigma\left(\left|x-x_{0}\right|, k T\right) \\
= & 2-2 e^{-\alpha\left(\left(x-x_{0}\right)^{2}+(\beta k T)^{2}\right)^{\kappa}} .
\end{aligned}
$$

In other words, for any point in time and space, the generalized distance between the approximated and the real value as seen by the sink is $\sqrt{\left(x-x_{0}\right)^{2}+(\beta k T)^{2}}$, and the corresponding distortion per field point of point $x$ as seen by the sink is given by $2(1-\sigma(\mid x-$ $\left.x_{0} \mid, k T\right)$ ).

In general, the statistics of the correlated data field might not be known, but they can be measured on-line, during the network deployment period (for instance, if the correlation is distance dependent, then nodes can make use of the distance information acquired from the neighbors for constructing routing tables, for the additional tasks of estimating the correlation structure and fitting the measurement parameters to a valid correlation model [2]). Also, note that the MSE error only perfectly characterizes the distortion for Gaussian signals

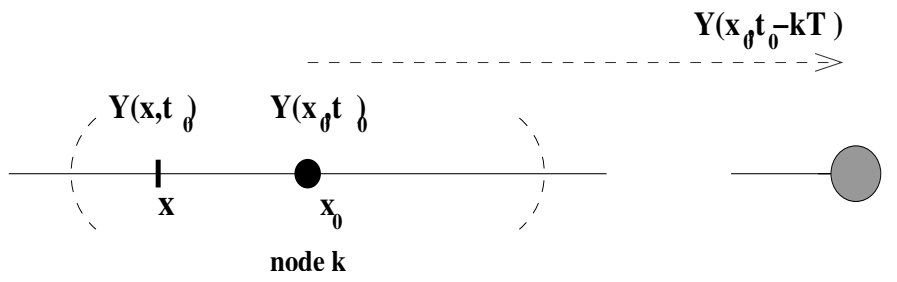

Fig. 3. The value at point $x$ is approximated by the value of the $k$ th sensor node placed at $x_{0}$, resulting in spatial distortion. Moreover, due to transmission over $k$ hops, the version that reaches the sink is delayed with $k T$, which results in time distortion. The combined result of the two distortion effects is the total distortion $D_{x}(N)$.

[1], thus a similar analysis for arbitrary spatio-temporal correlated fields should make use of alternative adequate distortion measures.

\section{Total Distortion}

We compute now the total distortion of the data estimated by the sink at a snapshot in time, in MSE sense. Consider node $k$, which is placed $k$ hops away from the sink. Denote the position of node $k$ as $x_{k}$, and the data that node $k$ measures at time $t$ as $Y\left(x_{k}, t\right)$. The data sent at time $t$ to the sink about the region $\left[x_{k}-d / 2, x_{k}+d / 2\right]$ is $Y\left(x_{k}, t\right)$, but since it is delayed with $k$ clock ticks, this packet actually reaches the sink at time $t+k T$ (see Fig. 3 ). In fact, at time $t$, the actual available data at the sink about node $k$ is $Y\left(x_{k}, t-k T\right)$. Thus, the corresponding distortion of reconstruction of the region covered by node $k$ is:

$$
D_{k}(N)=4 \int_{x=x_{k}}^{x_{k}+\frac{L}{2 N}}\left(1-\sigma\left(x-x_{k}, k T\right)\right) d x .
$$

For simplification, we can consider $x_{k}=0$ as axis origin for each node $k$, and then (3) becomes:

$$
D_{k}(N)=4 \int_{x=0}^{\frac{L}{2 N}}(1-\sigma(x, k T)) d x .
$$

The total distortion $D(N)$ is simply obtained by summing (4) over all nodes $k=0 \ldots N-1$ :

$$
D(N)=\sum_{k=0}^{N-1} 4 \int_{x=0}^{\frac{L}{2 N}}(1-\sigma(x, k T)) d x .
$$

Further, if we insert the correlation model for a Gaussian spatiotemporal process (1), we can finally write the total distortion of reconstruction of the whole field by the sink, as a function of $N$ :

$$
D(N)=\sum_{k=0}^{N-1} 4 \int_{x=0}^{\frac{L}{2 N}}\left(1-\exp \left(-\alpha\left(x^{2}+\beta^{2}(k T)^{2}\right)^{\kappa}\right)\right) d x .
$$

where $k$ counts the number of hops from a node to the sink, $T$ is the time delay per hop, $\beta$ is the time scaling constant, and $\alpha$ is a constant quantifying the intensity of correlation of the field. The term which is integrated is the distortion incurred by approximating the field between $\left[-\frac{L}{2 N}, \frac{L}{2 N}\right]$, around the node which is at $k$ hops away from the sink, with the value of that node delayed $k$ time steps.

The expression in (6) cannot be expressed in a closed form. However, an experimental analysis shows that (6) has always a minimum as a function of $N$. Moreover, in Section II-E, we will use a strong correlation approximation to derive in a closed-form the optimal value $N$ for which (6) is minimized. In general, the optimal value of $N$ is obtained by setting $\frac{\delta D(N)}{N}=0$ and numerically solving for $N$, by rounding the solution to the closest integer. 
Note that (2) completely expresses the distortion in MSE sense only for Gaussian random variables. Moreover, since spatially correlated Gaussian processes can only have certain structures for the correlation dependence on the distance [2], we will restrict our analysis to the models introduced in Section II-B.

\section{Optimum $N$ is Finite}

In this section we show that for the Gaussian correlation models introduced in Section II-B, there is indeed a finite optimum $N_{0}$ that minimizes (6). Denote:

$$
a_{n}=\sum_{k=0}^{n-1} \int_{x=0}^{\frac{L}{2 n}} \sigma(x, k T) d x
$$

Note that by definition $a_{n}$ is lower bounded by 0 . Thus, from (5) we can see that a sufficient condition for the existence of a finite optimum $N$ is that there exists $N_{0}$ such that for all $n>N_{0}, a_{n}$ is a decreasing sequence.

Correlation model: $\exp \left(-\alpha\left(x^{2}+\beta^{2}(k T)^{2}\right)\right)$

In this case, we can rewrite $a_{n}$ as

$$
a_{n}=\int_{x=0}^{\frac{L}{2 N}} e^{\alpha x^{2}} d x \cdot \sum_{k=0}^{n-1} \frac{1}{e^{\beta(k T)^{2}}} .
$$

But $\lim _{n \rightarrow \infty} a_{n} \searrow 0$, since the first term in the product converges to zero (the error function) and the second one can be easily shown to be upper bounded by a finite positive constant. Thus, for $\kappa=1$, the optimum $N$ that minimizes (6) is finite.

Correlation model: $\exp \left(-\alpha \sqrt{x^{2}+\beta^{2}(k T)^{2}}\right)$

This case is difficult to analyze analytically, due to the function that is integrated. However, our simulations in Section IV show that in this case too there is a finite optimal $N_{0}$.

\section{E. Strong Correlation Approximation}

In this section, we study the case when both $L / N$ and the time scale $\beta$ are small. In other words, data is strongly correlated both spatially and temporally. In this case, we can make the approximation:

$$
\begin{aligned}
1-e^{-\alpha\left(x^{2}+\beta^{2}(\beta k T)^{2}\right)^{\kappa}} & \approx 1-\left(1-\alpha\left(x^{2}+\beta^{2}(k T)^{2}\right)^{\kappa}\right) \\
& =\alpha\left(x^{2}+\beta^{2}(k T)^{2}\right)^{\kappa},
\end{aligned}
$$

which simplifies our analysis further.

Correlation model: $\exp \left(-\alpha\left(x^{2}+\beta^{2}(k T)^{2}\right)\right)$

First, we can write:

$$
\int_{x=0}^{\frac{L}{2 N}} \alpha\left(x^{2}+(\beta k T)^{2}\right) d x=\alpha\left(\frac{L^{3}}{24 N^{3}}+\frac{L(\beta k T)^{2}}{2 N}\right) .
$$

Further, from rewriting (6), it results:

$$
\begin{aligned}
D(N)= & \alpha\left(\frac{L^{3}}{2} \cdot \frac{1}{N^{2}}+\right. \\
& \left.+\frac{2 L(\beta T)^{2}}{3} \cdot N^{2}-L(\beta T)^{2} \cdot N+\frac{1}{3} L(\beta T)^{2}\right)
\end{aligned}
$$

Now we take the partial derivative of $D(N)$ with respect to $N$ and make it equal to zero. We obtain that $N_{0}$ is a solution of the equation:

$$
4 N^{4}-3 N^{3}-c=0
$$

where $c=\frac{L^{2}}{\beta^{2} T^{2}}$. For $c>0,(10)$ has a single positive solution $N_{0}$, which we plot in Fig. 4. This gives a good indication of the optimal value of $N_{0}$ as a function of $\frac{L^{2}}{\beta^{2} T^{2}}$; intuitively, $N_{0}$ increases with the decrease of the importance of delay in the distortion function, given by the time scale $\beta$.

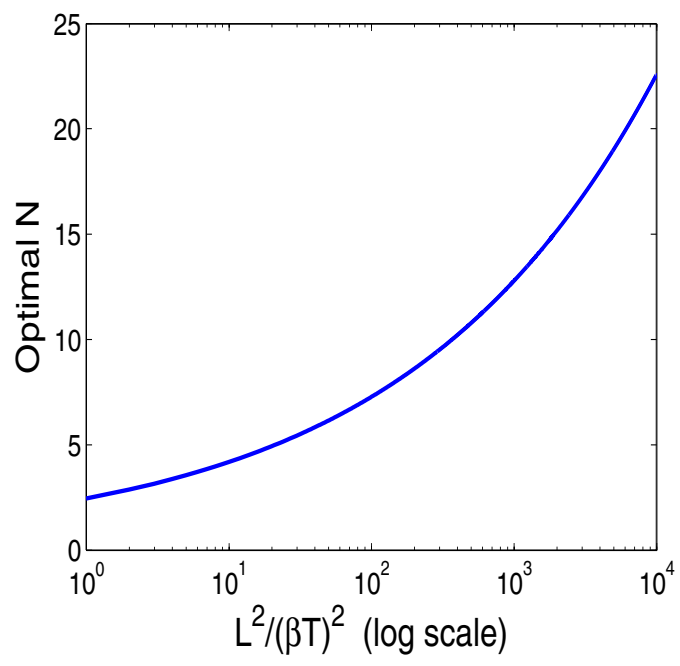

Fig. 4. Optimal value of $N$ as a function of $\frac{L^{2}}{\beta^{2} T^{2}}$, for $\kappa=1$, in the strong correlation approximation.

Note that, as expected, in the approximation of very strong correlation, the optimal $N$ does not depend on the value of $\alpha$ (which models the strength of correlation). The optimal value of $N$ only depends on the ratio between the length of the field $L$ and the time scaling parameter $\beta T$, that models the relative importance of delay in the distortion function as compared to spatial distortion.

Correlation model: $\exp \left(-\alpha \sqrt{x^{2}+\beta^{2}(k T)^{2}}\right)$

In this case, we compute:

$$
\begin{aligned}
& \int_{x=0}^{\frac{L}{2 N}} \alpha\left(x^{2}+(\beta k T)^{2}\right)^{\frac{1}{2}} d x= \\
= & -\frac{\alpha}{8 N}\left(-L \sqrt{\frac{L^{2}+4(\beta T k)^{2} N^{2}}{N^{2}}}+\right. \\
& +2(\beta T k)^{2} \ln (\beta T k)^{2} N+4(\beta T k)^{2} N \ln 2- \\
& \left.-4(\beta T k)^{2} N \ln \frac{L+\sqrt{\frac{L^{2}+4(\beta T k)^{2} N^{2}}{N^{2}}} N}{N}\right) .
\end{aligned}
$$

When $N$ is large, the second, third and fourth terms in the summation in the paranthesis cancel each other out, and it can be easily shown that (11) simplifies to

$$
\int_{x=0}^{\frac{L}{2 N}} \alpha\left(x^{2}+(\beta k T)^{2}\right)^{\frac{1}{2}} d x \approx \frac{\alpha L \beta T k}{4 N}
$$

By summing (12) over $k$, we can see that the resulting sum is a strictly increasing sequence in $N$. This only happens for $N$ large enough to guarantee the strong correlation approximation, however this is enough to show that the optimum $N_{0}$ has to be finite.

\section{Two-Dimensional MODEL}

\section{A. Total Distortion}

The case of a two-dimensional grid network (see Fig. 5) is studied similarly to the one-dimensional model. Consider a square area $L \times L$, on which $N^{2}$ nodes are uniformly placed on a square grid. The network is divided into Voronoi cells centered in the sensor nodes. We count the number of hops from each node to the sink on the most energy-efficient transmission structure for gathering uncorrelated data, which is the shortest path tree $(S P T)$. Note that, in general, since data at nodes are spatially correlated, the shortest 


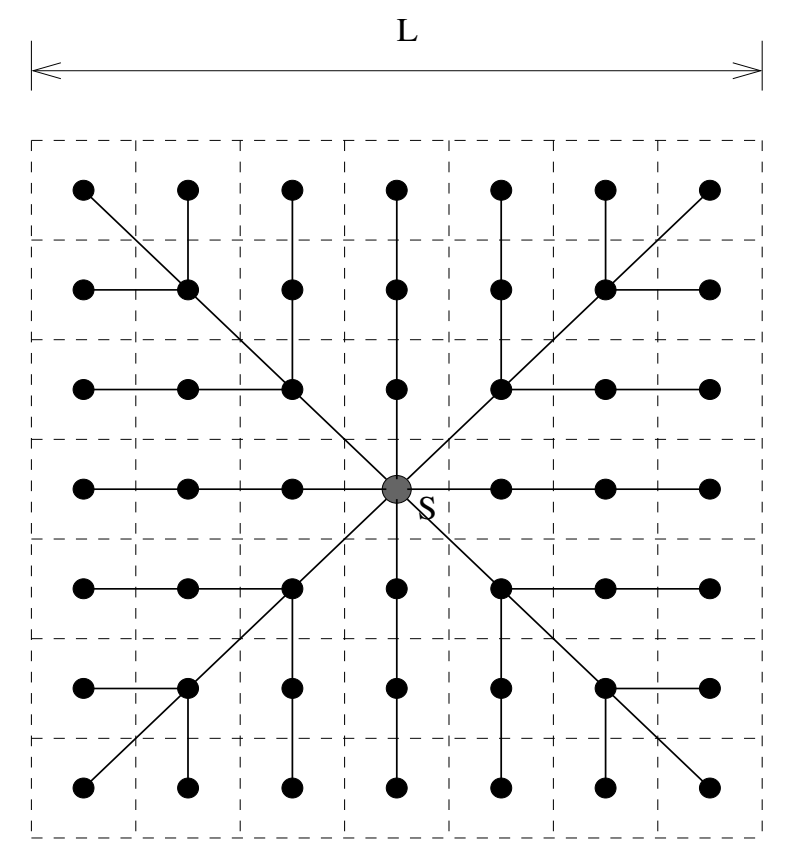

Fig. 5. A two-dimensional square grid. The Voronoi cell partition is drawn is dashed lines, and the shortest path tree $(S P T)$ is drawn in bold solid lines.

path tree is not the most energy-efficient transmission structure if in-network fusion by coding with side information is performed at nodes; moreover, finding the optimal transmission structure for such scenarios is NP-hard [3], [4]. Thus, in our analysis, we make the assumption that, due to limited resources, relay sensor nodes do not perform in-network fusion, namely they do not use as side information data from other nodes that use them as relay, to reduce the amount of data themselves need to transmit about their own measurements. In short, data is relayed without being processed.

In order to simplify the analysis, we consider a slightly modified setting for the two-dimensional square grid as compared to the onedimensional model (see Fig. 5). Namely, the modification from the one-dimensional study is that for the two-dimensional model we assume the sink gathers with no delay data in its corresponding Voronoi cell (in other words, the sink itself is considered as a regular sensor).

We plot in Fig. 5 the energy efficient paths from the nodes to the sink. Note that for every $k=0 \ldots N-1$ there are $8 k$ cells situated at $k$ hops away from the sink. Therefore, analogously to the one-dimensional case, we can write the total distortion for the two-dimensional case as:

$$
\begin{aligned}
D(N)= & \sum_{k=0}^{N-1} 4 \cdot 8 k \int_{x=0}^{\frac{L}{2 N}} \int_{y=0}^{\frac{L}{2 N}}\left(1-\exp \left(-\alpha\left(x^{2}+y^{2}+\right.\right.\right. \\
& \left.\left.\left.+\beta^{2}(k T)^{2}\right)^{\kappa}\right)\right) d y d x
\end{aligned}
$$

where $N$ is now the number of hops from the sink to the extremity of the square network.

\section{B. Strong Correlation Approximation}

In this section we use an approximation similar to the one in Section II-E, namely

$$
1-\exp \left(-\alpha\left(x^{2}+y^{2}+\beta^{2}(k T)^{2}\right)^{\kappa}\right) \approx \alpha\left(x^{2}+y^{2}+\beta^{2}(k T)^{2}\right)^{\kappa} .
$$

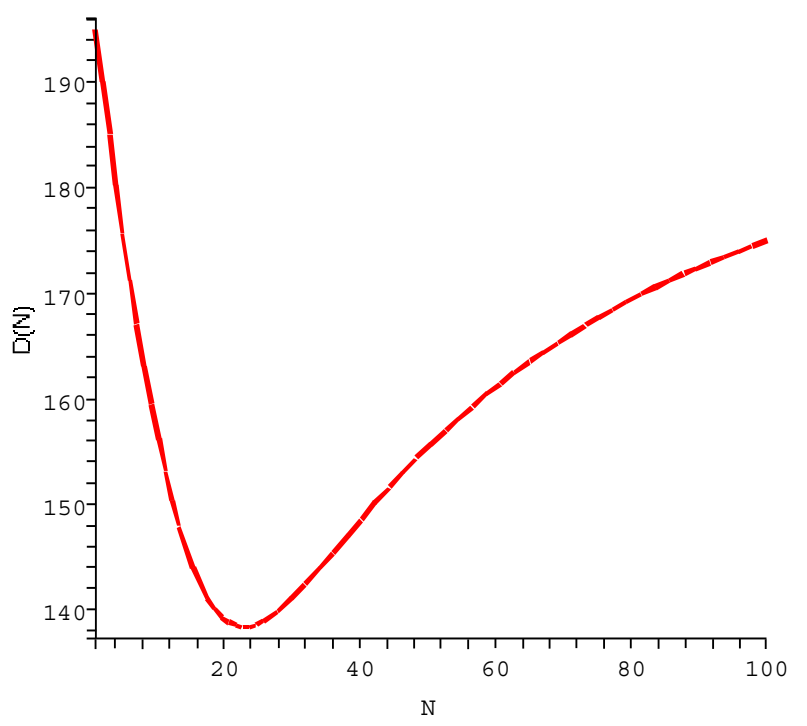

Fig. 6. Total estimation distortion of the field at the $\operatorname{sink} D(N)$ as a function of the number of nodes $N$, for a one-dimensional network; $\kappa=1$.

For the sake of simplicity, we analyze only the case $\kappa=1$, since the resulting optimization is easier. Namely, after some straightforward manipulations including taking the partial derivative of the resulting $D(N)$ with respect to $N$, we obtain that the optimal $N_{0}$ is a solution of the equation:

$$
N^{5}-N^{4}-\frac{c}{3} N+\frac{c}{2}=0
$$

where $c=\frac{L^{2}}{\beta^{2} T^{2}}$. A full analysis of the behavior of this polynomial is outside the scope of this paper. However, by numerical experiments, we are able to provide a set of insights:

- For $0<c<83.9$, this equation has no real positive solution (namely, it is strictly increasing and thus its optimum is attained at $N_{0}=1$, which means that in such a case the distortion caused by delay becomes so important that the optimal solution is to not place any sensor and let the sink estimate the whole field!)

- For $c \geq 83.9$, the equation has two positive real solutions, one $\left(N_{1} \in(1,2)\right)$ corresponding to a maximum of the function $D(N)$, and the other $N_{2}>2$ to a minimum. For $N>N_{2}$, $D(N)$ is strictly increasing. Thus, the optimum solution is either in $N_{0}=1$, or in $N_{2}$, both being finite integers.

\section{NumericAl Simulations}

In this section we do not use the approximation of strong correlation, but rather use the rough total distortion formulae given by (6) and (13).

We use Maple to plot in Fig. 6 the distortion $D(N)$ for the onedimensional case, as expressed in equation (6), as a function of $N$, for typical values of the constants involved: $\alpha=0.5$ (reasonable correlation decay), $\beta T=0.1$ (the constant scaling the time axis), $L=100$, and $\kappa=1$. In Fig. 7 we illustrate with a similar plot the case when $\kappa=0.5$, with $\alpha=0.05, \beta T=0.05, L=100$. We observe that, in general, there is an optimal $N$, that depends on the few constants involved in our model: $\alpha, \beta, \kappa, L$.

Fig. 8 shows the optimum values of $N$ that minimize (6) for various values of the correlation coefficients $\alpha$ and $\beta T(\kappa=1$, the length 


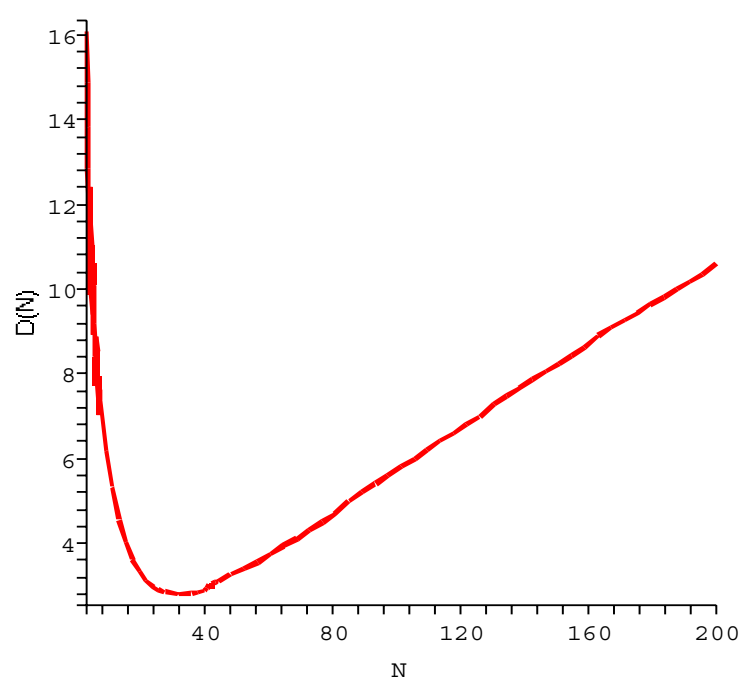

Fig. 7. Total estimation distortion of the field at the sink $D(N)$ as a function of the number of nodes $N$, for a one-dimensional network; $\kappa=0.5$.

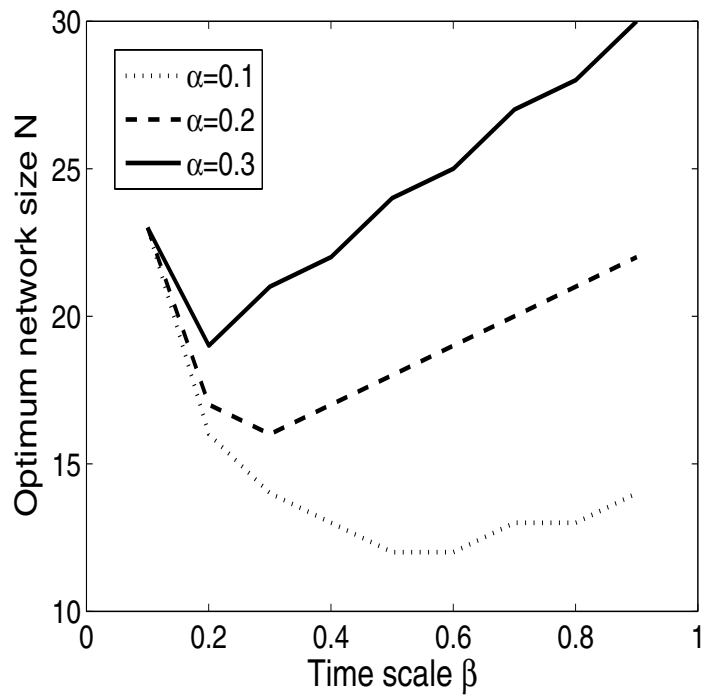

Fig. 8. One-dimensional grid: behavior of optimal number of sensors $N$ as a function of the correlation structure, for Gaussian processes with correlation $\exp \left(-\alpha\left(x^{2}+\beta(k T)^{2}\right)\right)$.

is $L=100$ ). We observe that for a given scaling $\beta$ for the time axis, the optimal value of $N$ decreases when the field becomes more correlated. Also, when the spatial correlation $\alpha$ is kept fixed, the optimal number of nodes $N$ depends on the time scaling constant $\beta$.

Finally, in Fig. 9, we plot the distortion $D(N)$ for the twodimensional case, as expressed in equation (13), as a function of $N$, for typical values of the constants involved: $\alpha=0.05, \beta=0.05$, $L=10$, and $\kappa=1$. We observe that again there is an optimal $N$ minimizing the total distortion. The ripples in the plots are due to Maple's graphical interpolation.

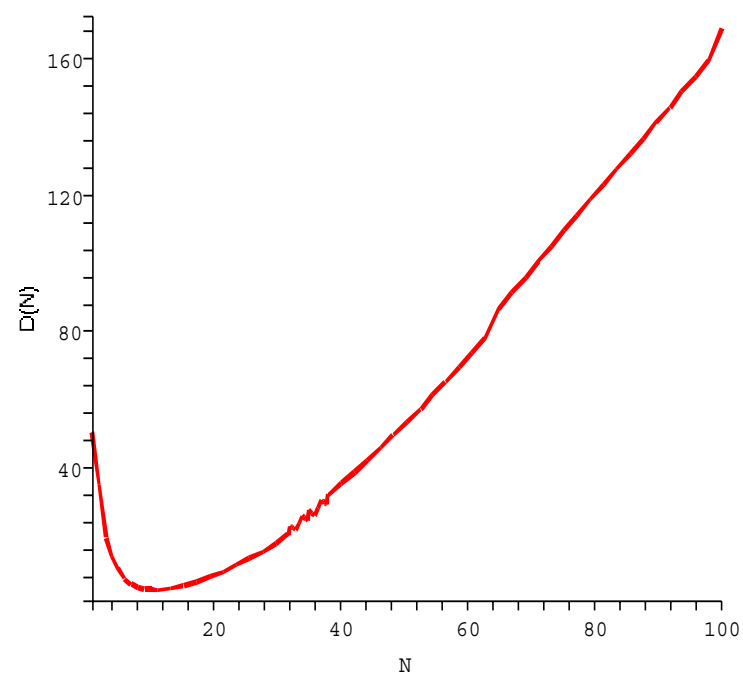

Fig. 9. Total estimation distortion of the field at the sink $D(N)$ as a function of the number of nodes $N$ for a two-dimensional network.

\section{CONCLUSIONS}

We considered real-time data gathering of spatio-temporally correlated processes by multihop sensor networks, where data is time critical and so delay results in suboptimal estimation at the sink. We considered two important issues in such a setting, namely power efficiency and delay. We showed that for Gaussian processes sampled by grid networks, there exists an optimal density of the network that minimizes the total distortion of reconstruction at the sink when real time reconstruction is required. We believe similar results hold for other spatio-temporally correlated processes, depending on their defining parameters. Our future work is focused on generalizing our results to networks with arbitrary network distributions. Also, we are investigating closed-loop system scenarios for distributed prediction and control with side information from the base station, where delay in transmitting the data through the network is a critical issue.

\section{REFERENCES}

[1] T.M. Cover, J.A. Thomas: Elements of Information Theory, John Wiley and Sons, Inc., 1991.

[2] N. Cressie: Statistics for Spatial Data, Wiley 1991.

[3] R. Cristescu, B. Beferull-Lozano, M. Vetterli, R. Wattenhofer: Network Correlated Data Gathering with Explicit Communication: NPCompleteness and Algorithms, submitted to IEEE/ACM Trans. on Networking, 2004.

[4] R. Cristescu, B. Beferull-Lozano, M. Vetterli: On Network Correlated Data Gathering, in Proc. INFOCOM 2004.

[5] D. Ganesan, S. Ratnasamy, H. Wang, D. Estrin: Coping with Irregular Spatio-Temporal Sampling in Sensor Networks, in Proc. HotNets-II, 2003.

[6] A. Goel, D. Estrin: Simultaneous Optimization for Concave Costs: Single Sink Aggregation or Single Source Buy-at-Bulk, ACM-SIAM Symposium on Discrete Algorithms, 2003.

[7] P. S. Maybeck, S. Peter: Stochastic Models, Estimation, and Control, Mathematics in Science and Engineering vol. 141, 1979.

[8] A. Kumar, P. Ishwar, K. Ramchandran: On Distributed Sampling of Bandlimited and Non-Bandlimited Sensor Fields, in Proc. ICASSP 2004.

[9] D. Marco, E. Duarte-Melo, M. Liu, D. L. Neuhoff: On the Many-toOne Transport Capacity of a Dense Wireless Sensor Network and the Compressibility of its Data, in Proc. IPSN 2003.

[10] G. J. Pottie, W. J. Kaiser: Wireless Integrated Sensor Networks, Communications of ACM 2000. 